\title{
BMJ Open Endovascular treatment for symptomatic intracranial artery stenosis: protocol for a systematic review and network meta- analysis
}

\author{
Tao Wang, ${ }^{1}$ Xue Wang, ${ }^{2}$ Kun Yang, ${ }^{3}$ Jing Zhang, ${ }^{4}$ Jichang Luo, ${ }^{1}$ Peng Gao, ${ }^{1}$ \\ Yan Ma, ${ }^{1}$ Liqun Jiao, ${ }^{1}$ Feng Ling ${ }^{1}$
}

To cite: Wang T, Wang $X$, Yang K, et al. Endovascular treatment for symptomatic intracranial artery stenosis: protocol for a systematic review and network meta-analysis. BMJ Open 2018;8:e022359. doi:10.1136/ bmjopen-2018-022359

- Prepublication history and additional material for this paper are available online. To view these files, please visit the journal online (http://dx.doi org/10.1136/bmjopen-2018022359).

TW and XW contributed equally.

Received 15 February 2018 Revised 27 March 2018 Accepted 6 June 2018

Check for updates

(c) Author(s) (or their employer(s)) 2018. Re-use permitted under CC BY-NC. No commercial re-use. See rights and permissions. Published by BMJ.

${ }^{1}$ Department of Neurosurgery, Xuanwu Hospital, Capital Medical University, Beijing, China

${ }^{2}$ Medical Library, Xuanwu Hospital, Capital Medical University, Beijing, UK

${ }^{3}$ Department of Evidence-Based Medicine, Xuanwu Hospital, Capital Medical University, Beijing, China

${ }^{4}$ Department of Neurology, Xuanwu Hospital, Capital Medical University, Beijing, China

Correspondence to

Dr Liqun Jiao; liqunjiao@sina.cn

\section{ABSTRACT}

Introduction Atherosclerotic intracranial artery stenosis

(ICAS) is one of most common causes of stroke, which is the second-leading cause of death worldwide. Medical, surgical and endovascular therapy are three major treatments for ICAS. Currently, medical therapy is considered as the standard of care for most patients with ICAS, while extracranial to intracranial bypass is only used in rare situations. Balloon angioplasty alone, balloon-mounted stent and self-expanding stent, collectively called endovascular treatment, have shown promising potentials in treating specific subgroups of patients with symptomatic ICAS; however, their comparative safety and efficacy is still unclear. Therefore, a systematic review with network meta-analysis is needed to establish a hierarchy of these endovascular treatments.

Methods and analysis The Preferred Reporting Items for Systematic Reviews and Meta-Analyses Protocols was followed to establish this protocol. The search will be limited to studies published from 1 January 2000 to the formal search date. Major databases including Cochrane Library, MEDLINE, EMBASE, Chinese Biomedical Literature Database, conference proceedings and grey literature database will be searched for clinical studies comparing at least two interventions for patients with symptomatic ICAS. Primary outcomes include short-term and long-term mortality or stroke rate. Random effects pairwise and network meta-analyses of included studies will be performed on STATA (V.14, StataCorp, 2015). The surface under the cumulative ranking curve and mean rank will be calculated in order to establish a hierarchy of the endovascular treatments. Evaluation of the risk of bias, heterogeneity, consistency, transitivity and quality of evidence will follow the recommendations of the Cochrane Handbook for Systematic Reviews of Interventions.

Ethics and dissemination Ethics approval is not needed as systematic review is based on published studies. Study findings will be presented at international conferences and published on a peer-reviewed journal.

PROSPERO registration number CRD42018084055; Pre-results.

\section{BACKGROUND}

\section{Description of the condition}

Stroke is currently the second-leading cause of death just behind ischaemic heart disease, causing 6.2 million deaths in 2015
Strengths and limitations of this study

- To the best of our knowledge, this study will be the first systematic review and network meta-analysis of safety and efficacy of three subtypes of endovascular treatment for patients with symptomatic intracranial stenosis.

- Besides randomised controlled studies, observational studies will also be included in order to obtain sufficient data for the network meta-analysis and improve the precision of estimates of adverse events.

- The present study has a clearly established aim, state of the art methods for data collection, quality evaluation and quantitative synthesis.

- The major challenge may come from unexpected heterogeneity from observational study designs. Stringent evaluation of transitivity will be conducted before data pooling for network meta-analysis.

worldwide. $^{1} 2$ Atherosclerotic intracranial artery stenosis (ICAS), one of most common causes of stroke, accounted for $10 \%-54 \%$ of all ischaemic strokes. Stroke mortality presented with regional variation, with a disproportionately high mortality in Asian countries, which might be partially attributable to higher prevalence of intracranial atherosclerosis in these regions. ${ }^{3}$ Great economic and family burden have been caused by stroke globally, especially in low/middle-income countries. ${ }^{4}$

\section{Description of the intervention}

Contemporary treatments for ICAS can be broadly categorised into medical, surgical and endovascular therapy. Currently, medical treatment remains the standard of care for patients with ICAS. $^{5}$ Aggressive medical management (ie, dual antiplatelet therapy along with intensive modifiable risk factor management) is supported by the latest studies $^{6-8}$ and recommended as the firstline therapy for symptomatic ICAS by the 
American Heart Association stroke prevention guidelines. ${ }^{9}$ Extracranial to intracranial bypass surgery (ECIC bypass) has been used as treatment for ICAS since the 1980s, but it was proven to be associated with a worse prognosis versus medical treatment for patients with ICAS in a randomised controlled trial (RCT) published in $1985 .{ }^{10}$ Ever since, EC-IC bypass is used in very few situations, such as stenoses progressing to occlusions with major haemodynamic impairment or in non-atherosclerotic lesions like moyamoya disease. ${ }^{11}$ Endovascular therapy, also called percutaneous transluminal angioplasty and stenting (PTAS), was adopted from management of coronary heart disease and the first cases of its use in ICAS were reported in the 1980 s. $^{12}$ It was considered as a minimally invasive approach to treat patients with symptomatic ICAS and was found to have an acceptable periprocedural complication rate and potential benefit in initial studies. ${ }^{8}{ }^{13} 14$ Although results of the Stenting versus Aggressive Medical Therapy for Intracranial Arterial Stenosis (SAMMPRIS) and the Vitesse Intracranial Stent Study for Ischemic Stroke Therapy (VISSIT) trials did not favour the use of PTAS in patients with ICAS, many neurovascular practitioners and academics still believe that there is a role for endovascular treatment of ICAS. ${ }^{15}$ Specific subgroups of patients, for example, African-American, Asian and Hispanic patients, ${ }^{16-19}$ are high-risk subgroup of patients who do not respond well to intensive medical treatment ${ }^{20}{ }^{21}$ and patients with hypoperfusion symptoms, ${ }^{21}$ which still needs to be confirmed by future studies.

\section{Rationale for the current systematic review}

Endovascular therapy can be generally divided into three subtypes: balloon angioplasty alone (BA), balloonmounted stent (BMS) or self-expanding stent (SES).$^{22}$ So far, none of them have been established to be the primary option of endovascular therapy for specific subgroups of patients with ICAS. Early studies comparing BA with stent placement showed comparable recurrent stroke or mortality rate, but stent treatment showed a lower rate of postoperative residual stenosis. ${ }^{23}{ }^{24}$ Comparable immediate procedural outcomes were reported by another study. ${ }^{25} \mathrm{~A}$ recent study, however, reported a significantly higher mortality $(17.6 \%$ vs $8.4 \%, \mathrm{p}<0.001)$ but no difference of iatrogenic stroke rate $(3.4 \%$ vs $3.6 \%, \mathrm{p}=0.826)$ in the BA group, compared with the stent group. ${ }^{26}$ Therefore, the safety and efficacy of BA versus stent placement is still unclear. As for the efficacy of BMS versus SES, the restenosis rate was showed to be higher in the SES than the BMS group. ${ }^{27-29}$ However, whether the other major complication rates are different between them still needs to be clarified. In summary, a systematic review with network meta-analysis that allows for both direct and indirect comparisons of multiple interventions is needed to decide the comparative effects of the three subtypes of endovascular therapy. To our knowledge, this kind of systematic review has not been previously completed.

\section{Objective}

The primary objectives of this study are to (1) determine both the safety and efficacy of different endovascular treatments (ie, BA, BMS or SES) on patients with symptomatic ICAS, and (2) establish a hierarchy of endovascular treatments for treating symptomatic ICAS, through a systematic review with network meta-analysis of randomised trials and observational studies.

\section{METHODS}

This protocol was developed according to the Preferred Reporting Items for Systematic Reviews and Meta-Analyses Protocols (PRISMA-P) (see online supplement 1, PRISMA-P Checklist). ${ }^{30}$ This systematic review has been perspectively registered on the PROSPERO database (CRD42018084055, available at http://www. crd.york.ac.uk/PROSPERO/display_record.php? ID= CRD42018084055). Any revision of this protocol and the whole review process will be updated timely on the PROSPERO registration. The conducting and reporting of this systematic review will follow the recommendations of the Cochrane Handbook for Systematic Reviews of Interventions and the PRISMA extension statement for reporting of systematic reviews incorporating network meta-analyses of healthcare interventions. ${ }^{3132}$

\section{Criteria for considering studies for this review \\ Types of studies}

RCTs and quasi-RCTs (non-blinded, interrupted time series) will be included. Observational cohort, casecontrol and registry studies will be included to obtain adequate statistical power, because rare outcomes will be included in our review and identifying these rare adverse events are important to assess the intervention safety, and RCTs lack adequate statistical power to evaluate these uncommon/rare safety outcomes due to type II (ie, false negative) error. ${ }^{33}$ Other types of studies including case series and case reports will be excluded. Studies published in Chinese journals will not be considered due to inappropriate randomisation procedures that have been reported in many of these studies. ${ }^{34}$

\section{Types of participants}

Patients with symptomatic ICAS and degree of stenosis more than $50 \%$ (verified by angiography) will be included. The stenosis is located in at least one major intracranial artery (intracranial internal carotid artery, vertebral artery or basilar artery and their major branches). Patients with ICAS with a transient ischaemic attack (TIA) or stroke are defined as symptomatic. A TIA was defined as a transient episode of neurological dysfunction (focal weakness or language disturbance, transient monocular blindness or required assistance in walking) caused by focal brain or retinal ischaemia that lasts for at least 10 min but resolves within 24 hours. ${ }^{35}$ ICAS related to the following factors will be excluded: arterial dissection, moyamoya disease, vasculitic disease, radiation-induced 
vasculopathy, fibromuscular dysplasia, sickle-cell disease, neurofibromatosis, suspected vasospastic process and suspected recanalised embolus.

\section{Types of interventions}

All competing interventions including any endovascular treatment as well as non-endovascular treatment strategy that can be administered for symptomatic ICAS are eligible for the analysis. Studies comparing at least two of the following eligible interventions will be considered in the analysis. We assume that any of the eligible interventions are, in principle, jointly randomisable among any patient that meets the inclusion criteria. If we identify any interventions that we are not aware of, we will consider them as eligible and include them in the network after assessing their comparability with those named below.

1. Interventions of direct interest

Studies that evaluated one or more of the following endovascular therapies namely BA, SES and BMS will be included. We will estimate the relative ranking of these interventions in the network meta-analysis according to primary outcomes.

2. Inclusion of additional interventions to supplement the analysis

Studies that evaluated non-endovascular treatment, namely medical treatment alone, and EC-IC bypass, will also be included to increase the amount of available (indirect) information in the analysis.

\section{Types of outcome measures}

Studies that reported at least one of the following outcomes will be included.

\section{Primary outcomes}

1. Short-term mortality or stroke rate (periprocedural or mean follow-up $\leq 3$ months).

2. Long-term mortality or stroke rate (mean follow-up $\geq$ 6 months).

\section{Secondary outcomes}

1. Long-term restenosis ( $\geq 50 \%$ stenosis verified by angiography, mean follow-up $\geq 6$ months).

2. TIA rate (short-term or long-term).

3. Other major complications.

\section{Search methods for identification of studies}

Literature search will mainly be executed in three databases: MEDLINE, EMBASE, the Cochrane Central Register of Controlled Trials. Search strategy has been drafted by an experienced librarian and revised by another librarian according to the Peer Review of Electronic Search Strategies checklist (see online supplement 2, Search strategy). ${ }^{36}$ The search will be limited to studies published from 1 January 2000 to the formal search date. In addition, we will also search other databases such as Chinese Biomedical Literature Database $(\mathrm{CBM})$, Web of Science and Open Grey, and conference proceedings for relevant abstracts, the ISRCTN registry (http://www.isrctn.com), government registries (http://www.clinicaltrials.gov) and WHO registries (http://www.who.int/trialsearch/) for ongoing and recently completed studies. There will be no restrictions on study type, language or publication type. We will search the bibliography of all included studies and request original data from the primary authors when necessary.

\section{Data collection and analysis \\ Selection of studies}

Two reviewers will independently complete the two levels of study screening and selection. In level one screening, reviewers will determine if a study is eligible for inclusion by screening the title and abstract of articles retrieved from the literature search. In level two screening, the full text of articles retained from level one screening will then be obtained and those that meet the eligible criteria will be included. When multiple studies report data from the same study population or multiple articles of the same study series are published in chronological order the study with the interventions of direct interest or the largest sample size will be retained. Before each level of screening, a pilot test, based on the predesigned test forms (see online supplement 3, Screening pilot-test form; adapted from Tricco $e t a l^{37}$ ), will be conducted to calculate inter-rater reliability and high agreement $(\geq 80 \%)$ is required to launch the formal screening. Discrepancies between the two reviewers will be resolved by discussion or, otherwise, a third reviewer. In cases of any ambiguity or insufficient data, study authors will be contacted for further information.

\section{Data extraction and management}

Similar with the screening process, data extraction will also be conducted by two reviewers, independently. A data abstraction form will be created in Excel and include two types of data:

1. Outcome data

Number of primary and secondary outcome events, total number of patients, the interventions being compared and follow-up duration will be extracted from included studies. Arm-level data will be extracted.

2. Data on potential effect modifiers

Data that may act as effect modifiers will be extracted from included studies, including: (1) study characteristics (eg, study design, volume of study centre, date of publication, journal of publication, study location(s), study funding); (2) population characteristics (eg, mean or median age, proportion of male patients, degree of preprocedural stenosis, functional status at presentation, medical history, drinking and smoking status, stenosis site of the intracranial artery); (3) intervention characteristics (eg, placement success rate, residual stenosis).

Also, a similar pilot test to calculate inter-rater reliability is required to confirm high agreement $(\geq 80 \%)$ between two reviewers. Similarly, two reviewers will be resolve disagreements by discussion or, otherwise, a third reviewer. 
And we will contact study authors for further information in case of any ambiguity or insufficient data.

\section{Assessment of risk of bias in included studies}

Similarly, two reviewers will independently assess risk of bias, and conflicts will be resolved through discussion or, otherwise, a third reviewer. The risk of bias of RCTs and quasi-RCTs will be assessed with items in the Cochrane Collaboration's tool, ${ }^{31}$ while that of non-RCTs (observational cohort and case-control studies) will be assessed with the Newcastle-Ottawa Scale (see online supplement 4, Newcastle-Ottawa Scale). ${ }^{38}$

\section{Measures of treatment effect}

As primary and secondary outcomes are all dichotomous data, ORs will be used as the measure of treatment effect. Relative treatment effects will be presented as the summary relative effect sizes (ORs) and associated 95\% credible intervals (CIs) for each possible pairwise comparison. Relative treatment ranking will also be estimated using the surface under the cumulative ranking curve (SUCRA) and mean ranks. ${ }^{39}$

\section{Dealing with missing data}

Some of the outcomes are assumed to be rare. Thus, zero events in one arm might be reported. In this case, 0.5 will be added to the numerator and one will be added to the denominator. Studies reporting zero events in all arms for primary outcomes will be excluded. ${ }^{40}{ }^{41}$ When encountering missing data in the included studies, we will contact the study authors for these data first. If the data are still unavailable on requests, we will impute missing data using established methods, including informative missing ORs for dichotomous outcomes and informative missingness difference of means for continuous outcomes. ${ }^{42}{ }^{43}$ Furthermore, a sensitivity analysis will be conducted to ensure that our imputations do not bias the final results. ${ }^{44}$

\section{Assessment of clinical and methodological heterogeneity and transitivity}

Across all eligible trials that compare each pair of interventions, descriptive statistics for potential effect modifiers described above (ie, study, population and intervention characteristics) will be generated. We will assess the presence of clinical and methodological heterogeneity both within and across treatment comparisons by calculating the $\mathrm{I}^{2}$ within each pairwise comparison. ${ }^{45}$ We will assess the assumption of transitivity across treatment comparisons by comparing the distribution of the potential effect modifiers across the different pairwise comparisons using boxplots or percentages. ${ }^{46} 47$ The above factors are ensured prior to conducting the following pairwise and network meta-analyses.

\section{Data synthesis}

As described above, if quantitative synthesis is not appropriate or the data are insufficient, the findings of our systematic review will be narratively reported. When

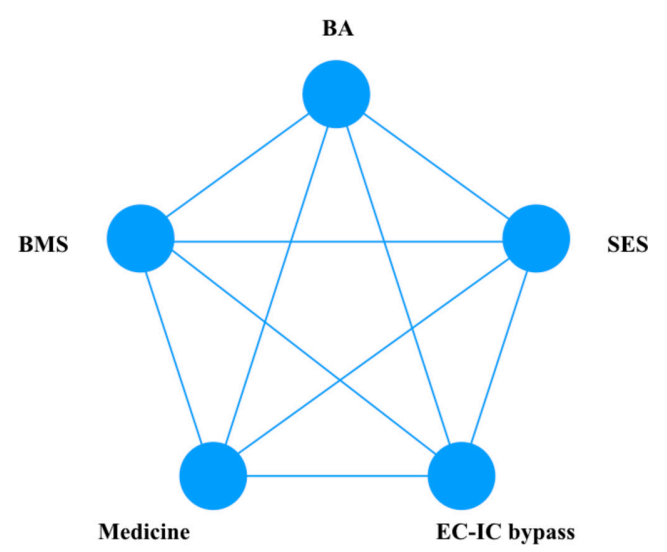

Figure 1 Network of all possible pairwise comparisons between the eligible interventions. BA, balloon angioplasty; BMS, balloon mounted stent; EC-IC bypass, extracranial to intracranial bypass; SES, self-expanding stent.

quantitative analysis is plausible, the following pairwise and network meta-analyses will be conducted in STATA (V.14, StataCorp, 2015). We will first restrict our analysis to RCTs, then include data from quasi-RCTs and, finally, data from observational studies. This sequential approach of analyses will provide an understanding of the contribution of each type of study design to our summary estimates.

\section{Methods for direct treatment comparisons}

Initially, we will perform standard pairwise meta-analyses for every direct treatment comparison with at least two studies (see figure 1). We will use the Bayesian random-effects models to derive summary effect measures with associated $95 \%$ CIs. ${ }^{48}$ The normal distribution will be used in the vague priors for all trial baselines, treatment effects and between-study SD.

\section{Methods for indirect and mixed comparisons}

We will perform network meta-analysis using the threelevel hierarchical, random-effects model as described in Schmitz et al. ${ }^{49}$ The normal distribution will also be used as the vague priors. We will rank relative treatment effects using mean ranks and the SUCRA. ${ }^{39}$ Rank-heat plots will be used to display the treatment rankings across multiple outcomes. ${ }^{50}$

\section{Assessment of statistical inconsistency}

We will evaluate the inconsistency between direct and indirect data locally by using the loop-specific method ${ }^{5152}$ and the node-splitting method, ${ }^{53}$ and globally by using the design-by-treatment interaction model. ${ }^{54}$

\section{Investigation of heterogeneity and inconsistency and sensitivity analyses}

Subgroup analyses will be conducted to explore if sufficient data are available. The following effect modifiers will be included in subgroup analyses: age, sex, degree of preprocedural stenosis, functional status at presentation and stenosis site of the intracranial artery. Network metaregression will be used to explore the effect of study 
year and study country if more than 10 studies are available. Sensitivity analyses will be conducted to test the robustness of our study findings by incorporating only data from the following studies when adequate studies are available: RCTs, quasi-RCTs and cohort studies reporting effect measures that are adjusted for important confounders.

\section{Patient and public involvement}

As the present study is a systematic review based on published data, patient and the public are not involved in the study design, conduct, data analysis and result dissemination.

\section{DISCUSSION}

The main anticipated challenge for the present systematic review and network meta-analysis is incorporating both randomised and observational studies. The rationale for including non-randomised studies is to obtain adequate statistical power to evaluate the outcomes, especially for the rare complications, because only a small amount of randomised studies were identified through an experimental search for eligible studies. Given that observational studies have inherited methodological limitations compared with randomised studies, another challenge is ensuring the treatment comparisons in our study maintain transitivity in our network meta-analyses while also remaining clinically meaningful to knowledge users.

It is expected that the study findings will address important questions about the relative safety and efficacy of different endovascular treatments for patients with symptomatic ICAS, allow patients and care providers to make informed decisions and provide comprehensive information for future study designs.

\section{Ethics and dissemination}

Ethics approval is not needed as systematic review is based on published studies. Study findings will be presented at international conferences and published on a peer-reviewed journal.

Contributors LJ, FL and YM developed the initial idea for this study. XW, TW and $K Y$ developed and revised the search strategy. TW, JZ and PG finished the study design. LJ, FL and YM were consulted about clinical issues. TW, JL and KY contributed to the original draft. PG, JZ and XW were responsible for the revision of the draft. TW and XW contributed equally to this article. All of the authors approved the final work prior to submission.

Funding This work was supported by the Ministry of Science and Technology of the People's Republic of China (2016YFC1301700).

Competing interests None declared.

Patient consent Not required.

Provenance and peer review Not commissioned; externally peer reviewed.

Data sharing statement Additional data from the study are available upon request to corresponding authors via e-mails.

Open access This is an open access article distributed in accordance with the Creative Commons Attribution Non Commercial (CC BY-NC 4.0) license, which permits others to distribute, remix, adapt, build upon this work non-commercially, and license their derivative works on different terms, provided the original work is properly cited, appropriate credit is given, any changes made indicated, and the use is non-commercial. See:@http://creativecommons.org/licenses/by-nc/4.0/.

\section{REFERENCES}

1. Banerjee C, Chimowitz MI. Stroke Caused by Atherosclerosis of the Major Intracranial Arteries. Circ Res 2017;120:502-13.

2. WHO. Global Health Observatory (GHO) data: WHO, 2017.

3. Johnston SC, Mendis S, Mathers CD. Global variation in stroke burden and mortality: estimates from monitoring, surveillance, and modelling. Lancet Neurol 2009;8:345-54.

4. Gretarsdottir S, Thorleifsson G, Manolescu A, et al. Risk variants for atrial fibrillation on chromosome 4 q25 associate with ischemic stroke. Ann Neurol 2008;64:402-9.

5. van den Wijngaard IR, Holswilder G, van Walderveen MA, et al. Treatment and imaging of intracranial atherosclerotic stenosis: current perspectives and future directions. Brain Behav 2016;6:e00536.

6. Derdeyn CP, Chimowitz MI, Lynn MJ, et al. Aggressive medical treatment with or without stenting in high-risk patients with intracranial artery stenosis (SAMMPRIS): the final results of a randomised trial. Lancet 2014;383:333-41.

7. Leung TW, Wang L, Soo YO, et al. Evolution of intracranial atherosclerotic disease under modern medical therapy. Ann Neurol 2015;77:478-86.

8. Zaidat OO, Fitzsimmons BF, Woodward BK, et al. Effect of a balloon-expandable intracranial stent vs medical therapy on risk of stroke in patients with symptomatic intracranial stenosis: the VISSIT randomized clinical trial. JAMA 2015;313:1240-8.

9. Kernan WN, Ovbiagele B, Black HR, et al. Guidelines for the prevention of stroke in patients with stroke and transient ischemic attack: a guideline for healthcare professionals from the American Heart Association/American Stroke Association. Stroke 2014;45:2160-236.

10. EC/IC Bypass Study Group. Failure of extracranial-intracranial arterial bypass to reduce the risk of ischemic stroke. Results of an international randomized trial. N Engl J Med 1985;313:1191-200.

11. Reith W, Berkefeld J, Dietrich P, et al. Diagnosis and Treatment of Intracranial Stenoses. Clin Neuroradiol 2015;25(Suppl 2):307-16.

12. Sundt TM, Smith HC, Campbell JK, et al. Transluminal angioplasty for basilar artery stenosis. Mayo Clin Proc 1980;55:673-80.

13. Wong KS, Chen C, Fu J, et al. Clopidogrel plus aspirin versus aspirin alone for reducing embolisation in patients with acute symptomatic cerebral or carotid artery stenosis (CLAIR study): a randomised, open-label, blinded-endpoint trial. Lancet Neurol 2010;9:489-97.

14. Turan TN, Cotsonis G, Lynn MJ, et al. Intracranial stenosis: impact of randomized trials on treatment preferences of US neurologists and neurointerventionists. Cerebrovasc Dis 2014;37:203-11.

15. Zaidat OO, Castonguay AC, Nguyen TN, et al. Impact of SAMMPRIS on the future of intracranial atherosclerotic disease management: polling results from the ICAD symposium at the International Stroke Conference. J Neurointerv Surg 2014;6:225-30.

16. Sacco RL, Kargman DE, Gu Q, et al. Race-ethnicity and determinants of intracranial atherosclerotic cerebral infarction. The Northern Manhattan Stroke Study. Stroke 1995;26:14-20.

17. Liu HM, Tu YK, Yip PK, et al. Evaluation of intracranial and extracranial carotid steno-occlusive diseases in Taiwan Chinese patients with MR angiography: preliminary experience. Stroke 1996;27:650-3.

18. White $H$, Boden-Albala $B$, Wang $C$, et al. Ischemic stroke subtype incidence among whites, blacks, and Hispanics: the Northern Manhattan Study. Circulation 2005;111:1327-31.

19. Wang $Y$, Zhao X, Liu L, et al. Prevalence and outcomes of symptomatic intracranial large artery stenoses and occlusions in China: the Chinese Intracranial Atherosclerosis (CICAS) Study. Stroke 2014;45:663-9.

20. Abou-Chebl A, Steinmetz H. Critique of "Stenting versus aggressive medical therapy for intracranial arterial stenosis" by Chimowitz et al in the new England Journal of Medicine. Stroke 2012;43:616-20.

21. Gao P, Zhao Z, Wang D, et al. China Angioplasty and Stenting for Symptomatic Intracranial Severe Stenosis (CASSISS): A new, prospective, multicenter, randomized controlled trial in China. Interv Neuroradiol 2015;21:196-204.

22. Teleb MS, Asif K, Castonguay AC, et al. Endovascular management of intracranial atherosclerosis. Neurosurg Clin N Am 2014;25:593-605.

23. Qureshi Al, Hussein HM, El-Gengaihy A, et al. Concurrent comparison of outcomes of primary angioplasty and of stent placement in high-risk patients with symptomatic intracranial stenosis. Neurosurgery 2008;62:1053-62. 
24. Siddiq F, Vazquez G, Memon MZ, et al. Comparison of primary angioplasty with stent placement for treating symptomatic intracranial atherosclerotic diseases: a multicenter study. Stroke 2008:39:2505-10.

25. Qureshi AI, Chaudhry SA, Siddiq F, et al. A randomized trial comparing primary angioplasty versus stent placement for symptomatic intracranial stenosis. J Vasc Interv Neurol 2013;6:34-41.

26. Villwock MR, Padalino DJ, Ramaswamy R, et al. Primary Angioplasty Versus Stenting for Endovascular Management of Intracranial Atherosclerotic Disease Following Acute Ischemic Stroke. J Vasc Interv Neurol 2016:9:1-6.

27. Yue $X$, Yin $Q, X i$ G, et al. Comparison of BMSs with SES for symptomatic intracranial disease of the middle cerebral artery stenosis. Cardiovasc Intervent Radiol 2011;34:54-60.

28. Park S, Kim JH, Kwak JK, et al. Intracranial stenting for severe symptomatic stenosis: self-expandable versus balloon-expandable stents. Interv Neuroradiol 2013;19:276-82.

29. Miao Z, Song L, Liebeskind DS, et al. Outcomes of tailored angioplasty and/or stenting for symptomatic intracranial atherosclerosis: a prospective cohort study after SAMMPRIS. $J$ Neurointerv Surg 2015;7:331-5.

30. Shamseer L, Moher D, Clarke M, et al. Preferred reporting items for systematic review and meta-analysis protocols (PRISMA-P) 2015 elaboration and explanation. BMJ 2015;350:g7647.

31. Higgins JPT GS. Cochrane Handbook for Systematic Reviews of Interventions Version 5.1.0 [updated March 2011]. The Cochrane Collaboration, 2011. http://handbook.cochrane.org/

32. Hutton B, Salanti G, Caldwell DM, et al. The PRISMA extension statement for reporting of systematic reviews incorporating network meta-analyses of health care interventions: checklist and explanations. Ann Intern Med 2015;162:777-84.

33. Eypasch E, Lefering R, Kum CK, et al. Probability of adverse events that have not yet occurred: a statistical reminder. BMJ 1995;311:619-20.

34. Wu TX LY, Liu GJ. Investigation of authenticity of 'claimed' randomized controlled trials (RCTs) and quality assessment of RCT reports published in China. Dublin, Ireland, 2006.

35. Easton JD, Saver JL, Albers GW, et al. Definition and evaluation of transient ischemic attack: a scientific statement for healthcare professionals from the American Heart Association/American Stroke Association Stroke Council; Council on Cardiovascular Surgery and Anesthesia; Council on Cardiovascular Radiology and Intervention; Council on Cardiovascular Nursing; and the Interdisciplinary Council on Peripheral Vascular Disease. The American Academy of Neurology affirms the value of this statement as an educational tool for neurologists. Stroke 2009;40:2276-93.

36. Sampson JB, Vardeny O, Flanigan KM. Aminoglycosides and other nonsense suppression therapies for the treatment of dystrophinopathy. Cochrane Database of Systematic Reviews 2009;64.

37. Tricco AC, Cogo E, Angeliki VA, et al. Comparative safety of antiepileptic drugs among infants and children exposed in utero or during breastfeeding: protocol for a systematic review and network meta-analysis. Syst Rev 2014;3:68.
38. Wells GA SB, O'Connell D, Peterson J, et al. The Newcastle-Ottawa Scale (NOS) for assessing the quality of nonrandomised studies in meta-analyses. $2010 \mathrm{http} / / / w w w . o h r i . c a / p r o g r a m s /$ clinical_ epidemiology/oxford.asp

39. Salanti G, Ades AE, loannidis JP. Graphical methods and numerical summaries for presenting results from multipletreatment meta-analysis: an overview and tutorial. J Clin Epidemiol $2011 ; 64: 163-71$

40. Sweeting MJ, Sutton AJ, Lambert PC. What to add to nothing? Use and avoidance of continuity corrections in meta-analysis of sparse data. Stat Med 2004;23:1351-75.

41. Bradburn MJ, Deeks JJ, Berlin JA, et al. Much ado about nothing: a comparison of the performance of meta-analytical methods with rare events. Stat Med 2007;26:53-77.

42. Spineli LM, Higgins JP, Cipriani A, et al. Evaluating the impact of imputations for missing participant outcome data in a network metaanalysis. Clin Trials 2013;10:378-88.

43. Mavridis D, White IR, Higgins JP, et al. Allowing for uncertainty due to missing continuous outcome data in pairwise and network metaanalysis. Stat Med 2015;34:721-41.

44. Carpenter J, Rücker G, Schwarzer G. Assessing the sensitivity of meta-analysis to selection bias: a multiple imputation approach. Biometrics 2011;67:1066-72.

45. Jackson D, Barrett JK, Rice S, et al. A design-by-treatment interaction model for network meta-analysis with random inconsistency effects. Stat Med 2014;33:3639-54.

46. Salanti G. Indirect and mixed-treatment comparison, network, or multiple-treatments meta-analysis: many names, many benefits, many concerns for the next generation evidence synthesis tool. Res Synth Methods 2012;3:80-97.

47. Jansen JP, Naci $\mathrm{H}$. Is network meta-analysis as valid as standard pairwise meta-analysis? It all depends on the distribution of effect modifiers. BMC Med 2013;11:159.

48. Sutton AJ, Abrams KR. Bayesian methods in meta-analysis and evidence synthesis. Stat Methods Med Res 2001;10:277-303.

49. Schmitz S, Adams R, Walsh C. Incorporating data from various trial designs into a mixed treatment comparison model. Stat Med 2013;32:2935-49.

50. Veroniki AA, Straus SE, Fyraridis A, et al. The rank-heat plot is a novel way to present the results from a network meta-analysis including multiple outcomes. J Clin Epidemiol 2016;76:193-9.

51. Song F, Altman DG, Glenny AM, et al. Validity of indirect comparison for estimating efficacy of competing interventions: empirical evidence from published meta-analyses. BMJ 2003;326:472.

52. Veroniki AA, Vasiliadis HS, Higgins JP, et al. Evaluation of inconsistency in networks of interventions. Int J Epidemiol 2013:42:332-45.

53. Dias S, Welton NJ, Caldwell DM, et al. Checking consistency in mixed treatment comparison meta-analysis. Stat Med 2010;29:932-44.

54. White IR, Barrett JK, Jackson D, et al. Consistency and inconsistency in network meta-analysis: model estimation using multivariate metaregression. Res Synth Methods 2012;3:111-25. 